\title{
New Euler-Maclaurin Expansions and Their Application to Quadrature Over the $s$-Dimensional Simplex
}

\author{
By Elise de Doncker*
}

\begin{abstract}
The $\mu$-panel offset trapezodial rule for noninteger values of $\mu$, is introduced in a one-dimensional context. An asymptotic series describing the error functional is derived. The values of $\mu$ for which this is an even Euler-Maclaurin expansion are determined, together with the conditions under which it terminates after a finite number of terms. This leads to a new variant of one-dimensional Romberg integration. The theory is then extended to quadrature over the $s$-dimensional simplex, the basic rules being obtained by an iterated use of one-dimensional rules. The application to Romberg integration is discussed, and it is shown how Romberg integration over the simplex has properties analogous to those for standard one-dimensional Romberg integration and Romberg integration over the hypercube.

Using extrapolation, quadrature rules for the s-simplex can be generated, and a set of formulas can be obtained which are the optimum so far discovered in the sense of requiring fewest function values to obtain a specific polynomial degree.
\end{abstract}

1. Introduction and Summary. The usual definition of the $\mu$-panel offset trapezoidal rule, as given in Lyness and Puri [4], restricts $\mu$ to being a positive integer. We extend their definition to apply with arbitrary positive $\mu$. An asymptotic expansion is derived, describing the error functional which corresponds to the rule sum. In this way it is, for example, revealed that the $\mu$-panel mid-point and end-point offset trapezoidal rules satisfy an even Euler-Maclaurin type expansion not only for integer but also for half-integer values of $\mu$. This suggests that one may use also the latter rules for one-dimensional Romberg integration. All of the one-dimensional results are presented in Section 2. Section 3 deals with the extension of these results to the $s$-dimensional unit simplex $\Delta_{s}$. This extension is carried out following the techniques of Lyness and Puri [4]. The s-dimensional rule is defined as an iterated rule operator, obtaining the rule sum by a repeated application of one-dimensional $\mu$-panel rules. If the product is formed using only mid-point or end-point offset trapezoidal rules, it is found to satisfy an even Euler-Maclaurin expansion for half-integer $\mu$ and for integer $\mu$, and can be used as a basic rule for Romberg extrapolation.

The extrapolation procedure for deriving rules of specified degree over $\Delta_{s}$ enjoys similar properties as standard Romberg integration and Romberg integration over the hypercube (Baker and Hodgson [1]). For the line and the hypercube the integration

Received June 13, 1978; revised November 28, 1978.

AMS (MOS) subject classifications (1970). Primary 65B15, 65D30; Secondary 65B05.

*This work is supported by the "Fonds voor Kollektief Fundamenteel Onderzoek" (FKFO, Belgium) under grant no. 2.0021.75. 
rules produced in this way are, however, far from optimum (in the sense of requiring fewest function values to obtain a specified polynomial degree). But for the simplex it will appear that rules can be generated which are the minimum point formulas yet known (Section 4). For their construction we employ mesh ratios which yield an even expansion of the error functional and which are as small as possible. With use of the sequence of mesh ratios $(k+1 / 2)_{k=0,1, \ldots}$ if $s$ is even, and the sequence $(k+1)_{k=0,1, \ldots}$ if $s$ is odd, simplex product mid-point rules are constructed which are invariant under the group of all affine transformations of $\Delta_{s}$ onto itself. The family constituted by the diagonal Romberg elements has also been constructed by Grundmann and Möller [2], using combinatorial methods. And they conjecture that, in the set of all integration rules of degree $d=2 p+1$ for $\Delta_{s}$, their formula has the smallest possible number of nodes if $s \geqslant d-1$. Special cases are the formula of degree $1(p=0)$ which is the mid-point formula $T_{s}: 1-1$ in Stroud [5], and the rule of degree $3(p=1)$ which coincides with the formula of Hammer and Stroud represented by $T_{s}: 3-1$ in Stroud [5].

\section{One-Dimensional Asymptotic Expansions.}

2.1. Basic Definitions and Terminology. Let $\phi$ be a real-valued function defined and integrable on the interval $[a, b]$. The integral of $\phi$ on $[a, b]$ will be denoted by

$$
I[a, b] \phi=\int_{a}^{b} \phi(x) d x
$$

or $I_{x}[a, b] \phi(x)$, if we want to indicate explicitly that the integration variable is $x$.

Definition (2.2). Let $\mu>0,0 \leqslant a \leqslant b \leqslant 1$ and $-1 \leqslant \alpha \leqslant 1$. The $\mu$-panel offset trapezoidal rule operator $R^{[\mu, \alpha]}[a, b]$ (also denoted by $R_{x}^{[\mu, \alpha]}[a, b]$ ) is defined by

$$
R^{[\mu, \alpha]}[a, b] \phi=\frac{1}{\mu} \sum_{j=-\infty}^{\infty} \theta_{j} \phi\left(x_{j}\right)
$$

with

$$
\begin{gathered}
x_{j}=\frac{j-1+t_{\alpha}}{\mu}, \\
t_{\alpha}=\frac{1+\alpha}{2}
\end{gathered}
$$

and

$$
\theta_{j}=H\left(b-x_{j}\right)-H\left(a-x_{j}\right),
$$

where $H$ represents Heaviside's function.

For $a \leqslant b,(2.5)$ has the meaning

$$
\begin{aligned}
\theta_{j}=1, & a<x_{j}<b, \\
1 / 2, & x_{j}=a<b \text { or } a<b=x_{j}, \\
0, & a=b, \\
0, & \text { otherwise. }
\end{aligned}
$$


In the case in which $\mu$ is an integer, $\mu=m$, Definition (2.2) coincides with the definition given by Lyness and Puri [4, pp. 274-275].

Note that

$$
R^{[\mu, 0]}[a, b] \phi=\frac{1}{\mu} \sum_{j=-\infty}^{\infty} G\left(\frac{2 j-1}{2 \mu}\right),
$$

with

$$
G(x)=[H(b-x)-H(a-x)] \phi(x) .
$$

Note also that the cases $\alpha=0$ and $\alpha=1$ with $\mu$ half-integer $(\mu=k+1 / 2, k \geqslant 0, k$ integer) turn out to be reflections of one another on $[0,1]$

$$
R^{[k+1 / 2,0]}[0,1] \phi \equiv R^{[k+1 / 2,1]}[0,1] \psi
$$

with

$$
\psi(x)=\phi(1-x)
$$

Figure (2.11) gives the abscissae in $[0,1]$ of the rules

$$
R^{[k+1 / 2,0]}[0,1] \phi=\frac{1}{k+1 / 2}\left[\sum_{j=1}^{k} \phi\left(\frac{2 j-1}{2 k+1}\right)+\frac{1}{2} \phi(1)\right]
$$

for $k=0,1,2$.

$$
R^{[1 / 2,0]}[0,1] \phi=\phi(1)
$$$$
R^{[3 / 2,0]}[0,1] \phi=\frac{2}{3}\left[\phi\left(\frac{1}{3}\right)+\frac{1}{2} \phi(1)\right]
$$$$
R^{[5 / 2,0]}[0,1] \phi=\frac{2}{5}\left[\phi\left(\frac{1}{5}\right)+\phi\left(\frac{3}{5}\right)+\frac{1}{2} \phi(1)\right]
$$

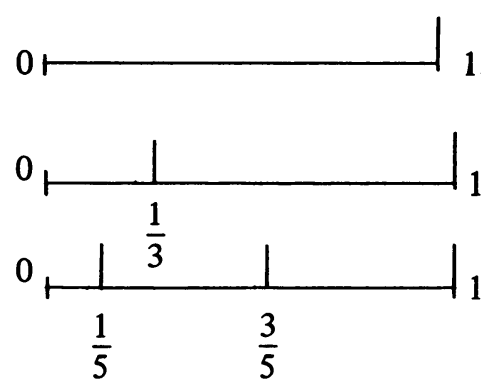

FigURE (2.11)

2.2. The Asymptotic Expansion for $R^{[\mu, \alpha]}[a, b] \phi$. The following theorem provides an asymptotic expansion for $R^{[\mu, \alpha]}[a, b] \phi$, which will be fundamental for deriving the expansion applying to the s-dimensional simplex (Section 3 ).

THEOREM (2.12). If the function $\phi:[a, b] \subseteq[0,1] \rightarrow \mathbf{R}$ is continuously differentiable of order $D+1$ on $[a, b]$

$$
\phi \in C^{D+1}[a, b] \text {, where } D \geqslant 0 \text { and } \mu>0 \text {, }
$$

the error functional expansion of $R^{[\mu, \alpha]}[a, b] \phi$ is given by 


$$
\begin{aligned}
R^{[\mu, \alpha]}[a, b] \phi-\int_{a}^{b} \phi(x) d x \\
=\sum_{q=1}^{D+1} \frac{1}{\mu^{q}}\left[\frac{\bar{B}_{q}\left(t_{\alpha}-\mu b\right)}{q !} \phi^{(q-1)}(b)-\frac{\bar{B}_{q}\left(t_{\alpha}-\mu a\right)}{q !} \phi^{(q-1)}(a)\right] \\
\quad-\frac{1}{\mu^{D+1}} \int_{a}^{b} \phi^{(D+1)}(t) \frac{\bar{B}_{D+1}\left(t_{\alpha}-\mu t\right)}{(D+1) !} d t,
\end{aligned}
$$

where the notation $\bar{B}_{q}(x)$ is used for the periodic Bernoulli functions as defined in Lyness [3].

Proof. The theorem has been proved for $\mu$ integer in Lyness [3] and is quoted in the above form but with $\mu$ replaced by $m$ in Lyness and Puri [4]. Thus, it holds for $\mu$ integer. Now, for the case where $\mu$ is noninteger, let $k$ be an integer, $k>\mu$, and define

$$
\widetilde{\phi}(x)=\phi(k x / \mu) .
$$

Then

$$
R^{[\mu, \alpha]}[a, b] \phi=\frac{k}{\mu} R^{[k, \alpha]}\left[\frac{\mu a}{k}, \frac{\mu b}{k}\right] \tilde{\phi} .
$$

Since $k>\mu$, we have

$$
\left[\frac{\mu a}{k}, \frac{\mu b}{k}\right] \subset[0,1]
$$

Since in addition $k$ is an integer, we may apply (2.12) to $R^{[k, \alpha]}[\mu a / k, \mu b / k] \widetilde{\phi}$ and hence derive the following expansion:

$$
\begin{aligned}
R^{[k, \alpha]}\left[\frac{\mu a}{k}, \frac{\mu b}{k}\right] \tilde{\phi}-I\left[\frac{\mu a}{k}, \frac{\mu b}{k}\right] \tilde{\phi} \\
=\sum_{q=1}^{D+1} \frac{1}{k^{q}}\left[\frac{\bar{B}_{q}\left(t_{\alpha}-\mu b\right)}{q !} \widetilde{\phi}^{(q-1)}\left(\frac{\mu b}{k}\right)-\frac{\bar{B}_{q}\left(t_{\alpha}-\mu a\right)}{q !} \widetilde{\phi}^{(q-1)}\left(\frac{\mu a}{k}\right)\right] \\
\quad-\frac{\mu}{k^{D+2}} \int_{a}^{b} \widetilde{\phi}^{(D+1)}\left(\frac{\mu t}{k}\right) \frac{\bar{B}_{D+1}\left(t_{\alpha}-\mu t\right)}{(D+1) !} d t .
\end{aligned}
$$

Multiplying both sides of (2.16) by $k / \mu$ and substituting

$$
\widetilde{\phi}^{(q-1)}\left(\frac{\mu t}{k}\right)=\left(\frac{k}{\mu}\right)^{q-1} \phi^{(q-1)}(t), \quad q=1, \ldots, D+2,
$$

yields the statement of Theorem (2.12) as written.

In order to establish some important properties of our summation formula (2.12), we write it in the form 


$$
R^{[\mu, \alpha]}[a, b] \phi=I[a, b] \phi+\sum_{q=1}^{D} \frac{A_{q}}{\mu^{q}}+E_{D+1},
$$

where

$$
A_{q}=A_{q}(\alpha, \mu, \phi, a, b)=\frac{\bar{B}_{q}\left(t_{\alpha}-\mu b\right)}{q !} \phi^{(q-1)}(b)-\frac{\bar{B}_{q}\left(t_{\alpha}-\mu a\right)}{q !} \phi^{(q-1)}(a),
$$

$$
q=1, \ldots, D
$$

and

$$
\begin{aligned}
E_{D+1} & =E_{D+1}(\alpha, \mu, \phi, a, b) \\
& =\frac{1}{\mu^{D+1}}\left[\frac{\bar{B}_{D+1}\left(t_{\alpha}-\mu b\right)}{(D+1) !} \phi^{(D)}(b)-\frac{\bar{B}_{D+1}\left(t_{\alpha}-\mu a\right)}{(D+1) !} \phi^{(D)}(a)\right. \\
& \left.\quad-\int_{a}^{b} \phi^{(D+1)}(t) \frac{\bar{B}_{D+1}\left(t_{\alpha}-\mu t\right)}{(D+1) !} d t\right] \\
& \sim O\left(\frac{1}{\mu^{D+1}}\right) .
\end{aligned}
$$

Inspection of the expressions (2.19) and (2.20) for $A_{q}$ and $E_{D+1}$, respectively, reveals immediately that the asymptotic series for $R^{[\mu, \alpha]}[a, b] \phi$ will terminate if $\phi$ is a polynomial.

COROLlary (2.21). If $\phi$ is a polynomial of degree $d$, then

$$
\begin{aligned}
A_{q}=0=E_{q}, \quad & \text { if } q>d+1, \\
& \text { or if } q>d, \mu a \text { and } \mu b \text { integer, } \\
\text { or if } q>d, \mu a \text { half-integer or integer and } \mu b \text { half- } & \text { integer or integer, } t_{\alpha}=0 \text { or } 1 / 2, \text { and } d \text { even. }
\end{aligned}
$$

If we consider the special and familiar case of the unit interval $(a=0$ and $b=1)$, (2.19) becomes

$$
A_{q}(\alpha, \mu, \phi)=\frac{\bar{B}_{q}\left(t_{\alpha}-\mu\right)}{q !} \phi^{(q-1)}(1)-\frac{\bar{B}_{q}\left(t_{\alpha}\right)}{q !} \phi^{(q-1)}(0), \quad q=1, \ldots, D .
$$

For use in one-dimensional Romberg integration, the most interesting cases will arise if $\alpha$ and $\mu$ are chosen so that $R^{[\mu, \alpha]}[0,1] \phi$ has an expansion in even inverse powers of $\mu$. From (2.22) we see that terms with $q$ odd in the expansion will drop out when both $\left\{t_{\alpha}-\mu\right\}$ and $\left\{t_{\alpha}\right\}$ are 0 or $1 / 2$ (where $\{x\}$ denotes the fractional part of $x$ ). This occurs when

$$
\{\mu\}=0 \quad \text { or } \quad 1 / 2
$$

and 


$$
t_{\alpha}=0 \text { or } 1 / 2
$$

Hence,

COROLlARY (2.25). If $\mu$ is integer or half-integer and $t_{\alpha}=0$ or $1 / 2$, $R^{[\mu, \alpha]}[0,1] \phi$ has an expansion (2.18) in even inverse powers of $\mu$.

The coefficients $A_{q}$ of the remaining terms depend on $\mu$ only through its fractional part $\{\mu\}$ (for $\phi$ and $\alpha$ fixed).

2.3. Application to One-Dimensional Romberg Integration. Since the coefficients $A_{q}$ in the expansion for $R^{[\mu, \alpha]}[0,1] \phi$, depend on $\mu$ only through its fractional part $\{\mu\}$, Romberg extrapolation can be applied to any sequence $\left(R^{\left[\mu_{k}, \alpha\right]}[0,1] \phi\right)_{k}$ computed with a sequence of mesh ratios $\left(\mu_{k}\right)_{k}$ satisfying

$$
\mu_{k}-\mu_{0}=\text { integer, } \quad k=0,1, \ldots
$$

The table is constructed in the usual way, setting

$$
T_{0}^{k}=R^{\left[\mu_{k}, \alpha\right]}[0,1] \phi
$$

and using the Neville algorithm to compute the other entries. Thus

$$
T_{p}^{k}=T_{p-1}^{k+1}+\mu_{k, p}\left(T_{p-1}^{k+1}-T_{p-1}^{k}\right), \quad p \geqslant 1,
$$

where when (2.27) has an even expansion,

$$
\mu_{k, p}=\mu_{k}^{2} /\left(\mu_{k+p}^{2}-\mu_{k}^{2}\right)
$$

and where

$$
\mu_{k, p}=\mu_{k} /\left(\mu_{k+p}-\mu_{k}\right)
$$

if the expansion is not known to be even.

Concerning the polynomial degree of accuracy of the elements occurring in the Romberg table, it follows from (2.21) that

COROLlARY (2.31). If the sequence $\left(R^{\left[\mu_{k}, \alpha\right]}[0,1] \phi\right)_{k}$ has an even expansion, then $T_{p}^{k}$ has polynomial degree of accuracy

$$
d=2 p+1, \text { if }\left(\mu_{k}\right)_{k} \text { is a sequence of integers, }
$$

$2 p, \quad$ if $\left(\mu_{k}\right)_{k}$ is a sequence of half-integers.

If $\left(R^{\left[\mu_{k}, \alpha\right]}[0,1] \phi\right)_{k}$ has an expansion which is not even, then $T_{p}^{k}$ has polynomial degree of accuracy $d=p-1$.

\section{Asymptotic Expansions Applying to the s-Dimensional Simplex.}

3.1. Product Trapezoidal Rules for the s-Dimensional Simplex. In this section we define an $s$-dimensional analogue, for the $s$-simplex, of the one-dimensional trapezoidal rule operator $R^{[\mu, \alpha]}[0,1]$, and construct an Euler-Maclaurin-like expansion for the error functional. We rely on the derivation of Lyness and Puri [4]. 
NEW EULER-MACLAURIN EXPANSIONS

1009

We extend their theory, which is restricted to positive integer mesh ratios $\mu$, by allowing arbitrary positive values of $\mu$. Let us consider the s-dimensional unit simplex

$$
\Delta_{s}=\left\{\left(x_{1}, \ldots, x_{s}\right) \mid i=1, \ldots, s: x_{i} \geqslant 0, \sum_{i=1}^{s} x_{i} \leqslant 1\right\}
$$

and a real-valued function $\phi$ defined and integrable over $\Delta_{s}$.

The integral of $\phi$ over $\Delta_{s}$ can be expressed in the form of an iterated integral

$$
\begin{aligned}
I \Delta_{s} \phi & =\int_{\Delta_{s}} \phi\left(x_{1}, \ldots, x_{s}\right) d x_{1} \cdots d x_{s} \\
=I_{x_{1}}[0,1] I_{x_{2}}\left[0,1-x_{1}\right] I_{x_{3}}\left[0,1-x_{1}-x_{2}\right] & \\
\cdots & I_{x_{s}}\left[0,1-\sum_{i=1}^{s-1} x_{i}\right] \phi\left(x_{1}, \ldots, x_{s}\right),
\end{aligned}
$$

where the subscripts $x_{i}, i=1, \ldots, s$, indicate the order of the integration variables. Permutations of the integration variables will produce different integration orders, and result in integration over some other simplex.

In a corresponding manner we define the $s$-dimensional simplex product trapezoidal rule operator.

Definition (3.3). Let $\mu>0$ and $-1 \leqslant \alpha_{i} \leqslant 1$ for all $i=1, \ldots, s$. We define

$$
R^{[\mu, \vec{\alpha}]} \Delta_{s} \phi=R_{x_{1}}^{\left[\mu, \alpha_{1}\right]}[0,1] R_{x_{2}}^{\left[\mu, \alpha_{2}\right]}\left[0,1-x_{1}\right]
$$

$$
\cdots R_{x_{s}}^{\left[\mu, \alpha_{s}\right]}\left[0,1-\sum_{i=1}^{s-1} x_{i}\right] \phi\left(x_{1}, \ldots, x_{s}\right),
$$

where each $R_{x_{i}}^{\left[\mu, \alpha_{i}\right]}, i=1, \ldots, s$, represents a one-dimensional offset trapezoidal rule defined as in the previous section and operating in the $\dot{x}_{i}$-direction.

Three-dimensional examples include

$$
\begin{aligned}
& R^{[1, \overrightarrow{0}]} \Delta_{3} \phi=0, \\
& R^{[2, \overrightarrow{0}]} \Delta_{3} \phi=\frac{1}{8} \phi\left(\frac{1}{4}, \frac{1}{4}, \frac{1}{4}\right), \\
& R^{[3, \overrightarrow{0}]} \Delta_{3} \phi=\frac{1}{27}\left[\phi\left(\frac{1}{6}, \frac{1}{6}, \frac{1}{6}\right)+\phi\left(\frac{1}{6}, \frac{1}{6}, \frac{3}{6}\right)+\phi\left(\frac{1}{6}, \frac{3}{6}, \frac{1}{6}\right)+\phi\left(\frac{3}{6}, \frac{1}{6}, \frac{1}{6}\right)\right],
\end{aligned}
$$

$$
\begin{aligned}
R^{[1 / 2, \overrightarrow{0}]} \Delta_{3} & =0 \\
R^{[3 / 2, \overrightarrow{0}]} \Delta_{3} & =\frac{8}{27}\left[\frac{1}{2} \phi\left(\frac{1}{3}, \frac{1}{3}, \frac{1}{3}\right)\right], \\
R^{[5 / 2, \overrightarrow{0}]} \Delta_{3}= & \frac{8}{125}\left[\phi\left(\frac{1}{5}, \frac{1}{5}, \frac{1}{5}\right)+\frac{1}{2} \phi\left(\frac{3}{5}, \frac{1}{5}, \frac{1}{5}\right)+\frac{1}{2} \phi\left(\frac{1}{5}, \frac{3}{5}, \frac{1}{5}\right)\right. \\
& \left.+\frac{1}{2} \phi\left(\frac{1}{5}, \frac{1}{5}, \frac{3}{5}\right)\right]
\end{aligned}
$$


It will be interesting to note that some of these rules have the property of being invariant under the group of all affine transformations of $\Delta_{s}$ onto itself. The following can be established in a straightforward manner:

THEOREM (3.5). The rules $R^{[\mu, \overrightarrow{0}]} \Delta_{s}$ with $\mu$ half-integer, if $s$ is even, and $\mu$ integer, if $s$ is odd, are affine invariant with respect to $\Delta_{s}$.

3.2. The Asymptotic Expansion for $R^{[\mu, \vec{\alpha}]} \Delta_{s} \phi$. We shall now establish an asymptotic expansion for $R^{[\mu, \vec{\alpha}]} \Delta_{s} \phi$ having the same form as its one-dimensional analogue (2.18). In Lyness and Puri [4] it is proved that, for integer mesh ratios $\mu=m$, an expansion in inverse powers of $m$ is obtained, where the coefficients are independent of $m$. For noninteger $\mu, R^{[\mu, \vec{\alpha}]} \Delta_{s} \phi$ will be shown to have an expansion in inverse powers of $\mu$, where in general the coefficients depend on $\mu$. As we want to establish the sdimensional analogues of corollaries (2.21), (2.25) and (2.31), we need to determine the nature of this dependence. However, the derivation of the asymptotic expansion will not be given in full, since it can be constructed following the general lines of the three-dimensional proof given in Lyness and Puri [4].

THEOREM (3.6). Let the function $\phi: \Delta_{s} \rightarrow \mathbf{R}$ be continuously differentiable of order $D+1$ on $\Delta_{s}$

$$
\phi \in C^{D+1} \Delta_{s}, \text { where } D \geqslant 0
$$

(requiring that $\phi$ and all its partial derivatives of total order $D+1$ or less, be continuous in all variables, within and on the boundary of $\Delta_{s}$ ). Then $R^{[\mu, \vec{\alpha}]} \Delta_{s} \phi$ satisfies an Euler-Maclaurin-like expansion

$$
R^{[\mu, \vec{\alpha}]} \Delta_{s} \phi=I \Delta_{s} \phi+\sum_{q=1}^{D} \frac{A_{q}}{\mu^{q}}+E_{D+1},
$$

with

$$
A_{q}=A_{q}(\vec{\alpha}, \mu, \phi), \quad q=1, \ldots, D, \quad E_{D+1}=E_{D+1}(\vec{\alpha}, \mu, \phi)
$$

The dependence of $A_{q}$ on $\mu$ ( $\phi$ and $\vec{\alpha}$ fixed) is only through the fractional part of $\mu$. Furthermore,

$$
\left|E_{D+1}(\vec{\alpha}, \mu, \phi)\right| \sim O\left(\frac{1}{\mu^{D+1}}\right) .
$$

The proof of this theorem is long, and is of a similar structure to that of the proof of Theorem 4.29 given by Lyness and Puri [4, pp. 279-282]. Here we merely state the construction of the coefficients $A_{q}$. Using this the reader will have no difficulty in adapting the proof in Lyness and Puri [4] to establish the theorem.

3.3. Construction and Properties of the Coefficients $A_{q}$. Set

$$
a\left(x_{1}, \ldots, x_{s}\right)=\phi\left(x_{1}, \ldots, x_{s}\right)
$$

and define recursively the following functions, for $k=0,1, \ldots, s-1$ : 


$$
\begin{aligned}
a_{q_{s-k}, \ldots, q_{s}}\left(x_{1}, \ldots, x_{s-k-1}\right) & \frac{\bar{B}_{q_{s-k}}\left(-\mu+\sum_{i=1}^{s-k} t_{\alpha_{i}}\right)}{q_{s-k} !} \frac{\partial^{q_{s-k-1}}}{\partial x_{s-k}^{q_{s-k-1}}} \\
= & \left.\cdot a_{q_{s-k+1}, \ldots, q_{s}}\left(x_{1}, \ldots, x_{s-k}\right)\right|_{x_{s-k}=1-\sum_{i=1}^{s-k-1} x_{i}} \\
& -\left.\frac{\bar{B}_{q_{s-k}\left(t_{\alpha_{s-k}}\right)} \frac{\partial^{q_{s-k}-1}}{\partial x_{s-k}^{q_{s-k}-1}}}{} \quad a_{q_{s-k+1}, \ldots, q_{s}}\left(x_{1}, \ldots, x_{s-k}\right)\right|_{x_{s-k}=0}, q_{s-k} \geqslant 1 .
\end{aligned}
$$

If $q_{s-k}=0$, this is interpreted as an integral

$$
\begin{aligned}
& a_{0, q_{s-k+1}, \ldots, q_{s}}\left(x_{1}, \ldots, x_{s-k-1}\right) \\
& =\int_{0}^{1-\sum_{i=1}^{s-k-1} x_{i}} a_{q_{s-k+1}, \ldots, q_{s}}\left(x_{1}, \ldots, x_{s-k}\right) d x_{s-k} .
\end{aligned}
$$

With $k=s-1$, the constants $a_{q_{1}, \ldots, q_{s}}$ are defined. The coefficients $A_{q}$ are now obtained as

$$
A_{q}=\sum_{\Sigma_{i=1}^{s} q_{i}=q} a_{q_{1}, \ldots, q_{s}} .
$$

From (3.9) we see that each $a_{q_{1}, \ldots, q_{s}}$ is a sum of terms involving the product of a $\phi$-depending factor multiplied by $\prod_{i=1}^{s} \bar{B}_{q_{i}}\left(\lambda_{i}\right)$, where the Bernoulli functions are evaluated either at $\lambda_{i}=t_{\alpha_{i}}$ or $\lambda_{i}$ of the form

$$
\lambda_{i}=-\mu+\sum_{l=1}^{i} t_{\alpha_{l}} .
$$

It follows immediately that the dependence of $A_{q}$ on $\mu$ ( $\phi$ and $\vec{\alpha}$ fixed) is only through its fractional part $\{\mu\}$.

From the expression for $A_{q}$ in (3.11) we see that $q$ odd corresponds to the occurrence of at least one odd subscript in each $a_{q_{1}, \ldots, q_{s}}$. So if each of the quantities $t_{\alpha_{i}}$ and $-\mu+\Sigma_{l=1}^{i} t_{\alpha_{l}}$, for $i=1, \ldots, s$, is integer or half-integer, this will imply that $A_{q}$ with $q$ odd vanishes. This occurs when

$$
\{\mu\}=0 \text { or } 1 / 2
$$

and

$$
t_{\alpha_{i}}=0 \text { or } 1 / 2, \quad i=1, \ldots, s .
$$


Hence, we have

COROLlaRY (3.15). If $\mu$ is integer or half-integer and $t_{\alpha_{i}}=0$ or $1 / 2$ for $i=$ $1, \ldots, s$, then $R^{[\mu, \vec{\alpha}]} \Delta_{s} \phi$ has an asymptotic expansion (3.6) in even inverse powers of $\mu$. The coefficients $A_{q}$ of the remaining terms depend on $\mu$ only through its fractional part $\{\mu\}$ (for $\phi$ and $\vec{\alpha}$ fixed).

We shall now prove that the asymptotic series (3.6) terminates if $\phi$ is a polynomial, which will establish its possible application to the construction of $s$-dimensional quadrature rules.

Lemma (3.16). If $\phi$ is a polynomial of degree $d$, then

$$
\begin{gathered}
a_{q_{1}, \ldots, q_{s}}=0, \text { if } \mu \text { integer and } \sum_{i=1}^{s} q_{i}>d+s-1, \\
\text { or } \mu \text { arbitrary and } \sum_{i=1}^{s} q_{i}>d+s .
\end{gathered}
$$

Proof. If the function $a_{q_{s-k+1}, \ldots, q_{s}}\left(x_{1}, \ldots, x_{s-k}\right)$ defined in (3.8), (3.9) and (3.10) is a polynomial of degree $d_{s-k}$ it follows that $a_{q_{s-k}, \ldots, q_{s}}\left(x_{1}, \ldots, x_{s-k-1}\right)$ is a polynomial of degree

$$
d_{s-k-1} \leqslant d_{s-k}-q_{s-k}+1 .
$$

Applying (3.17) $s-1$ times shows that $a_{q_{2}, \ldots, q_{s}}\left(x_{1}\right)$ is of degree

$$
d_{1} \leqslant d-\sum_{i=2}^{s} q_{i}+s-1 \text {. }
$$

If $\mu$ is integer, then for $k=s-1(3.9)$ and (3.10) reduce to

$$
a_{q_{1}, \ldots, q_{s}}=\frac{\bar{B}_{q_{1}}\left(t_{\alpha_{1}}\right)}{q_{1} !} \int_{0}^{1} \frac{\partial^{q_{1}}}{\partial x_{1}^{q_{1}}} a_{q_{2}, \ldots, q_{s}}\left(x_{1}\right) d x_{1} .
$$

So, if in this case

$$
d-\sum_{i=2}^{s} q_{i}+s-1<q_{1}
$$

i.e.

$$
d+s-1<\sum_{i=1}^{s} q_{i}
$$

then the integrand in (3.19) is identically zero, and

$$
a_{q_{1}, \ldots, q_{s}}=0
$$

which proves the lemma for integer $\mu$.

If $\mu$ is not integer, we apply (3.17) $s$ times, yielding that $a_{q_{1}, \ldots, q_{s}}$ has degree

$$
d_{0} \leqslant d-\sum_{i=1}^{s} q_{i}+s
$$


So, if

$$
d-\sum_{i=1}^{s} q_{i}+s<0
$$

i.e.

$$
d+s<\sum_{i=1}^{s} q_{i}
$$

then $a_{q_{1}, \ldots, q_{s}}$ becomes a polynomial of negative degree, which assumes the meaning that $a_{q_{1}, \ldots, q_{s}}$ is identically zero. This completes the proof.

From (3.11) we see that $A_{q}$ is a sum of coefficients $a_{q_{1}, \ldots, q_{s}}$ with $\Sigma_{i=1}^{s} q_{i}=q$. This leads to the following theorem:

THEOREM (3.24). If $\phi$ is a polynomial of degree $d$, then

$$
\begin{aligned}
& A_{q}=0, \text { if } q>d+s, \\
& \quad \text { or if } q>d+s-1 \text { and } \mu \text { integer, } \\
& \quad \text { or if } q>d+s-1 \text { and } \mu \text { half-integer, } t_{\alpha_{i}}=0 \text { or } 1 / 2 \text { for } i=1, \ldots, s, \\
& \text { and } d+s-1 \text { even. }
\end{aligned}
$$

3.4. Application to s-Dimensional Romberg Integration. The conditions under which certain coefficients $A_{q}$ vanish are given in (3.15) and (3.24). They are fundamental for the determination of the polynomial degree of accuracy of the elements in the Romberg table. We consider a sequence of rules $\left(R^{\left[\mu_{k}, \vec{\alpha}\right]} \Delta_{s} \phi\right)_{k}$, where the mesh ratios $\left(\mu_{k}\right)_{k}$ satisfy (2.26).

THEOREM (3.25). If $\left(R^{\left[\mu_{k}, \vec{\alpha}\right]} \Delta_{s} \phi\right)_{k}$ has an even expansion, then $T_{p}^{k}$ has polynomial degree of accuracy

$$
\begin{aligned}
d=2 p+2-s, & \text { if }\left(\mu_{k}\right)_{k} \text { is a sequence of integers, } \\
& 2 p+1-s, \quad \text { if }\left(\mu_{k}\right)_{k} \text { is a sequence of half-integers. }
\end{aligned}
$$

If $\left(R^{\left[\mu_{k}, \vec{\alpha}\right]} \Delta_{s} \phi\right)_{k}$ has an expansion which is not even, then $T_{p}^{k}$ has polynomial degree of accuracy $d=p-s$.

\section{Particular Sets of Integration Rules for $\Delta_{s}$, Generated by Means of Romberg} Extrapolation. The element $T_{p}^{k}$ of the Romberg table is a specified linear combination of the elements $T_{0}^{j}, j=k, k+1, \ldots, k+p$. Each element $T_{0}^{j}$ is a weighted sum of function values. Thus, each element $T_{p}^{k}$ may be expressed directly as a weighted sum of function values-or as a quadrature rule. This rule depends on the values of the members of the sequence $\mu_{k}, \mu_{k+1}, \ldots, \mu_{k+p}$ and on the formula used to define $T_{0}^{j}, j=k, k+1, \ldots, k+p$.

Carrying out this unscrambling process in the case of the line or the hypercube has not usually led to any interesting insight into the nature of the quadrature rules. In the case of the simplex, some of the results of doing this are of interest. 
Definition (4.1). The quadrature rule $J_{p}\left(\mu_{0}, \Delta_{s}\right)$ is the rule obtained by setting

$$
T_{0}^{k}=R^{\left[\mu_{k}, \overrightarrow{0}\right]} \Delta_{s} \phi
$$

using mesh ratios

$$
\mu_{k}=\mu_{0}+k, \quad k=0,1, \ldots,
$$

and calculating the element $T_{p}^{0}$.

We shall be interested in the polynomial degree of this rule and the number of function values required by it, in the cases when $\mu_{0}=1$ or $1 / 2$ (in which cases $T_{0}^{k}$ has an even expansion).

The polynomial degree $d_{p}\left(\mu_{0}, \Delta_{s}\right)$ of $J_{p}\left(\mu_{0}, \Delta_{s}\right)$ follows immediately from Theorem (3.25).

\section{THEOREM (4.3).}

$$
\begin{aligned}
& J_{p}\left(1 / 2, \Delta_{s}\right) \text { is of polynomial degree } d_{p}\left(1 / 2, \Delta_{s}\right)=2 p+1-s, \\
& J_{p}\left(1, \Delta_{s}\right) \text { has polynomial degree } d_{p}\left(1, \Delta_{s}\right)=2 p+2-s .
\end{aligned}
$$

It must be noted that the rules $J_{p}\left(\mu_{0}, \Delta_{s}\right)$ for which

$$
d_{p}\left(\mu_{0}, \Delta_{s}\right)<0
$$

coincide with zero diagonal elements $T_{p}^{0}$.

Let us consider, for each $s$, the formulas

$$
J_{p}\left(1 / 2, \Delta_{s}\right), \text { if } s \text { is even, } J_{p}\left(1, \Delta_{s}\right), \quad \text { if } s \text { is odd. }
$$

These rules are constructed as linear combinations of basic rules $R^{[\mu, \overrightarrow{0}]} \Delta_{s}$ which are affine invariant following Theorem (3.5). Hence,

THEOREM (4.6). The rules $J_{p}\left(1 / 2, \Delta_{s}\right)$ for $s$ even and $J_{p}\left(1, \Delta_{s}\right)$ for $s$ odd are invariant under the group of all affine transformations of $\Delta_{s}$ onto itself.

A bound on the number of integration points $\nu_{p}\left(\mu_{0}, \Delta_{s}\right)$ used by $J_{p}\left(\mu_{0}, \Delta_{s}\right)$ can be computed by summing the numbers of points needed by its basic rules $R^{\left[\mu_{k}, \vec{\alpha}\right]} \Delta_{s}, k=0,1, \ldots, p$. This bound is equal to the total number of such points if the basic rules have no common points. We have

THEOREM (4.7). The number of integration points of $J_{p}\left(\mu_{0}, \Delta_{s}\right)$ satisfies

$$
\nu_{p}\left(\mu_{0}, \Delta_{s}\right) \leqslant \sum_{j=0}^{p^{\prime}}\left(\begin{array}{c}
s+j \\
s
\end{array}\right)=\left(\begin{array}{c}
s+p^{\prime}+1 \\
p^{\prime}
\end{array}\right) \text {, }
$$

where $p^{\prime}$ is given by the following table

\begin{tabular}{c|cc}
$p^{\prime}$ & $s$ odd & s even \\
\hline$\mu_{0}=\frac{1}{2}$ & $p-\frac{s-1}{2}$ & $p-\frac{s}{2}$ \\
$\mu_{0}=1$ & $p-\frac{s-1}{2}$ & $p-\frac{s}{2}+1$
\end{tabular}


Note that the number of points is related to the polynomial degree of the formula by

$$
p^{\prime}=\left[1 / 2 d_{p}\left(\mu_{0}, \Delta_{s}\right)\right]
$$

(where the notation $[x]$ is used to indicate the largest integer not exceeding $x$ ).

Table (4.10) gives the polynomial degree $\left(d_{p}\right)$ and the number of points $\left(\nu_{p}\right)$ of the rules $J_{p}\left(\mu_{0}, \Delta_{s}\right)$ for $\mu_{0}=1 / 2$ and for $\mu_{0}=1, p=0,1,2,3,4$ and $s=1,2,3$, 4. The affine invariant rules are indicated by $A$. Some of these rules are well known. The rules of degree 1 coincide with the mid-point formulas $T_{s}: 1-1$, and the rules of degree 3 correspond to the formulas $T_{s}: 3-1$ in Stroud [5]. These are known to be minimum point formulas (the former for $s \geqslant 1$ and the latter for $s \geqslant 2$ ), in the sense of requiring fewest function values to attain a specified polynomial degree. The entire set of rules which are indicated to be affine invariant has recently been found by Grundmann and Möller [2]. They conjecture that, considering all the rules of degree $d=2 p^{\prime}+1$ for $\Delta_{s}$, the rule belonging to their family has the smallest possible number of nodes if $s \geqslant d-1$.

We have demonstrated that the rules $J_{p}\left(1 / 2, \Delta_{s}\right)$ and $J_{p}\left(1, \Delta_{s}\right)$ can be generated by means of Romberg extrapolation in a straightforward way. Yet it may be interesting to see some examples of these rules. We have listed rules of polynomial degree $\leqslant 5$, for $s=1,2,3,4$ in Table (4.11). In this table we make use of the notation $\Sigma \phi\left(x_{1}, \ldots, x_{s}\right)_{s}$ to indicate that the summation runs over the set of points which includes $\left(x_{i_{1}}, \ldots, x_{i_{s}}\right)$ if $\left(i_{1}, \ldots, i_{s}\right)$ is a permutation of $(1, \ldots, s)$.

\section{TABLE (4.10a)}

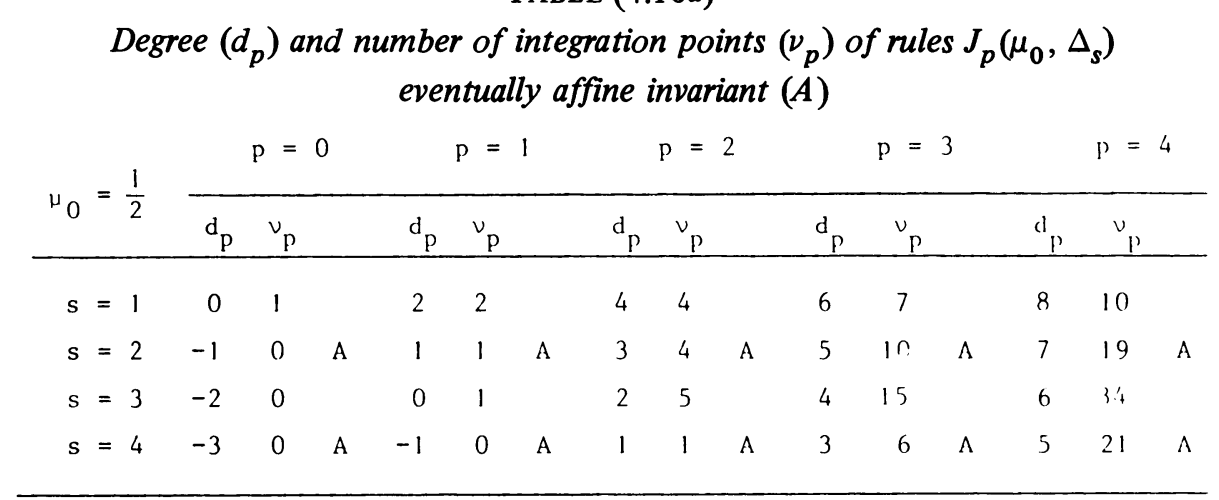

TABLE (4.10b)

\begin{tabular}{|c|c|c|c|c|c|c|c|c|c|c|c|c|c|c|c|c|}
\hline \multirow{2}{*}{${ }^{\prime} 0$} & \multirow{2}{*}{$=1$} & \multicolumn{3}{|c|}{$p=0$} & \multicolumn{3}{|c|}{$p=1$} & \multicolumn{3}{|c|}{$p=2$} & \multicolumn{3}{|c|}{$p=3$} & \multicolumn{3}{|c|}{$p=4$} \\
\hline & & $\mathrm{d}_{\mathrm{p}}$ & $v_{\mathrm{P}}$ & & $\mathrm{d}_{\mathrm{p}}$ & $v_{p}$ & & $d_{p}$ & $v_{p}$ & & $d_{p}$ & $u_{p}$ & & $\mathrm{~d}_{\mathrm{p}}$ & $v^{v}$ & \\
\hline $\mathbf{s}$ & $=1$ & 1 & 1 & A & 3 & 3 & $\Lambda$ & 5 & 5 & $A$ & 7 & 9 & $\Lambda$ & 9 & 13 & $\wedge$ \\
\hline $\mathrm{S}$ & $=2$ & 0 & 1 & & 2 & 4 & & 4 & 9 & & 6 & 19 & & 8 & 33 & \\
\hline $\mathbf{s}$ & $=3$ & -1 & 0 & A & 1 & 1 & A & 3 & 5 & A & 5 & 15 & A & 7 & 35 & $\Lambda$ \\
\hline $\mathrm{s}$ & $=4$ & -2 & 0 & & 0 & 1 & & 2 & 6 & & 4 & 21 & & 6 & 56 & \\
\hline
\end{tabular}




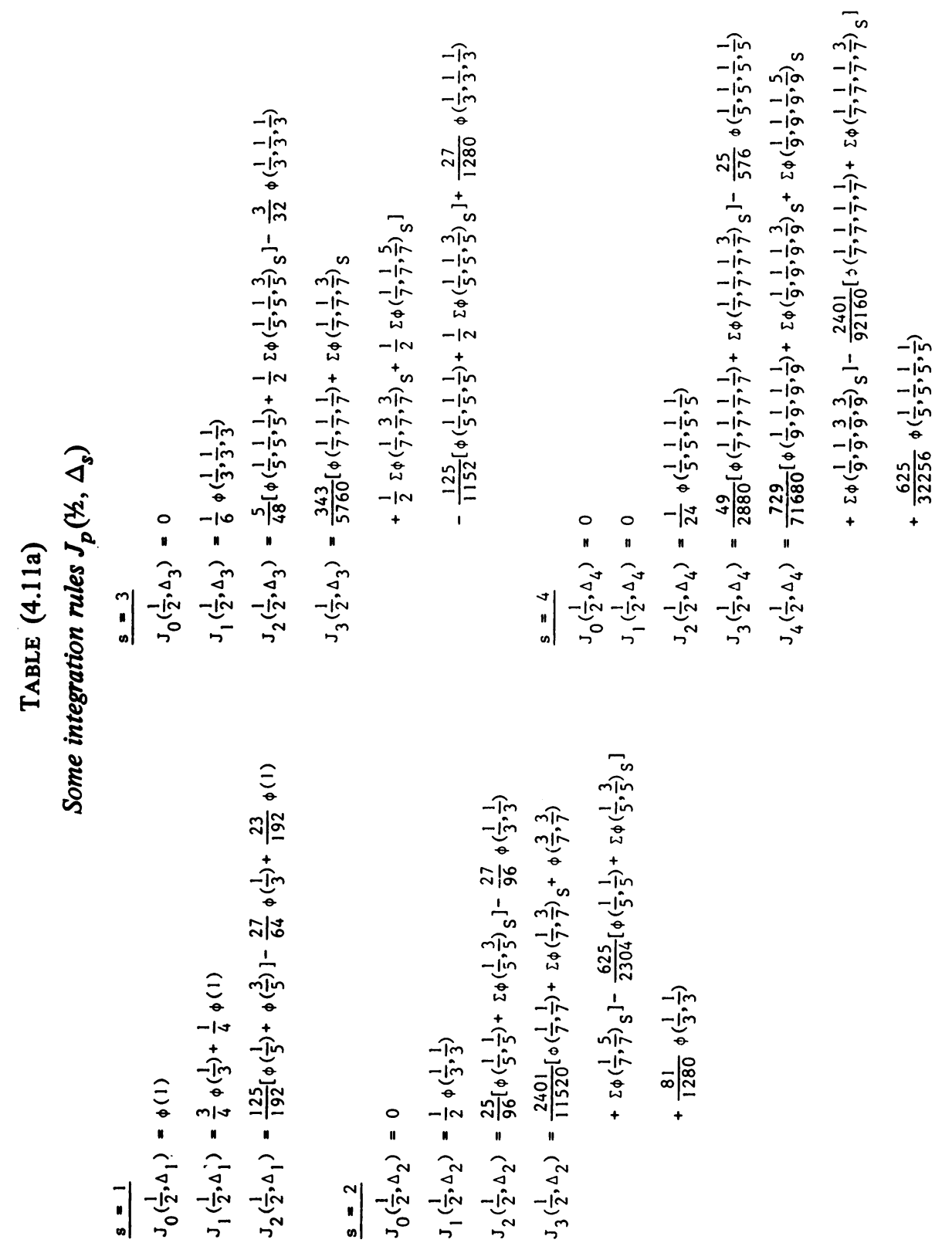




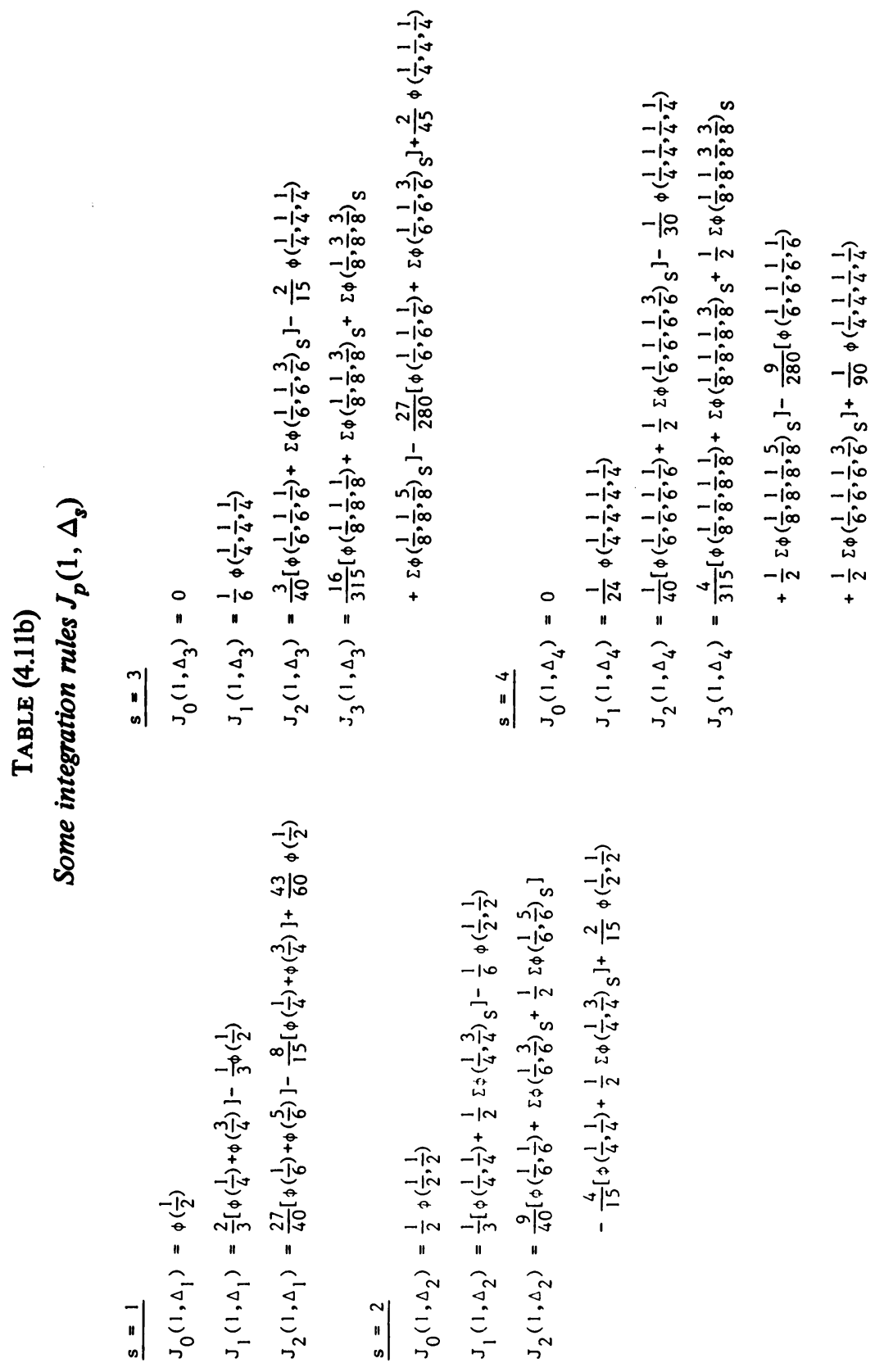


5. Conclusion. This paper gives a generalization of one-dimensional Romberg integration, as it introduces the use of noninteger mesh ratios. In the same sense it extends the work by Lyness and Puri [4], where a basis for Romberg integration over the $s$-dimensional simplex is established. Moreover, we have demonstrated that, for the simplex, the procedure of generating quadrature formulas by unscrambling Romberg table elements can be used to produce sets of rules which contain minimum point formulas and formulas which are the optimum so far discovered, in the sense of requiring fewest function values to obtain a specific polynomial degree.

Acknowledgement. I am very grateful to Dr. J. N. Lyness for his valuable suggestions.

Applied Mathematics and Programming Division K. U. L.

University of Leuven

Celestijnenlaan 200A

B-3030 Heverlee, Belgium

1. C. T. H. BAKER \& G. S. HODGSON, "Asymptotic expansions for integration formulas in one or more dimensions," SIAM J. Numer. Anal., v. 8, 1971, pp. 473-480.

2. A. GRUNDMANN \& H. M. MÖLLER, "Invariant integration formulas for the $n$ simplex by combinatorial methods," SIAM J. Numer. Anal., v. 15, 1978, pp. 282-290.

3. J. N. LYNESS, "Quadrature over a simplex: Part 1. A representation of the integrand function," SIAM J. Numer. Anal., v. 15, 1978, pp. $122-133$.

4. J. N. LYNESS \& K. K. PURI, "The Euler-Maclaurin expansion for the simplex," Math. Comp., v. 27, 1973, pp. 273-293.

5. A. H. STROUD, Approximate Calculation of Multiple Integrals, Prentice-Hall, Englewood Cliffs, N. J., 1971. 